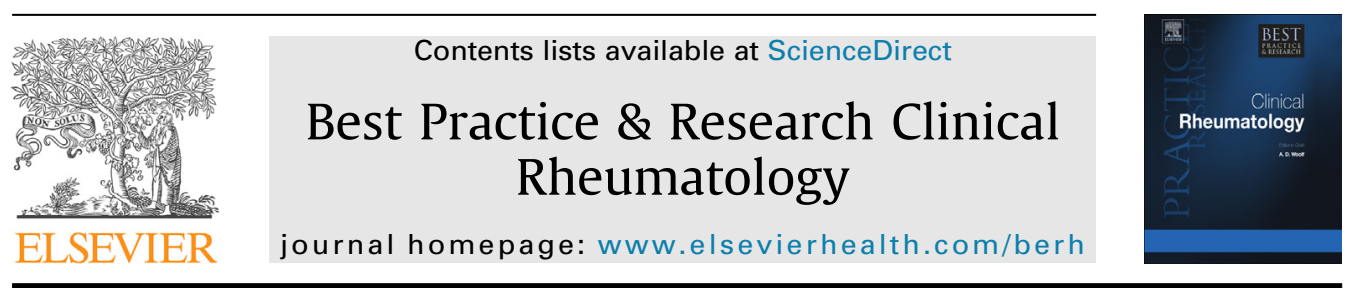

5

\title{
Pain in systemic connective tissue diseases
}

\author{
Manuela Di Franco ${ }^{a, *, 1}$, Laura Bazzichi ${ }^{\text {b, }}{ }^{\text {, Roberto Casale }}{ }^{\text {, }}$, \\ Piercarlo Sarzi-Puttini ${ }^{\text {, }}$, Fabiola Atzeni ${ }^{\text {e }}$ \\ ${ }^{\text {a }}$ Rheumatology Unit, Department of Internal Medicine and Medical Specialities, Sapienza University of \\ Rome, Italy \\ ${ }^{\mathrm{b}}$ Rheumatology Unit, Department of Clinical and Experimental Medicine, University of Pisa, Italy \\ ${ }^{\mathrm{c}}$ Department of Clinical Neurophysiology and Pain Rehabilitation Unit (RC), Foundation Salvatore Maugeri \\ IRCCS, Montescano, Italy \\ ${ }^{\mathrm{d}}$ Rheumatology Unit, L.Sacco University Hospital, Milan, Italy \\ e IRCCS Galeazzi Orthopedic Institute, Milan, Italy
}

\section{Keywords:}

Connective tissue diseases

Inflammatory pain

Chronic pain

SLE

SSc

\begin{abstract}
A B S T R A C T
Pain is frequent in patients with connective tissue diseases (CTDs), particularly those affected by systemic sclerosis (SSc) and systemic lupus erythematosus (SLE) in which it is virtually ubiquitous and can have different causes. The SLE classification criteria include pain associated with musculoskeletal involvement, which are frequently the initial symptom of SLE and can include arthralgia, arthritis and/or myalgia. Chronic widespread pain, the cornerstone of fibromyalgia (FM), is also frequently associated with CTDs.

Chronic pain has a considerable impact on mental health, and the professional and family lives of patients. It can be due to many disorders, but there are few reports concerning its prevalence during the course of other diseases.

It is essential to identify the origin of pain in CTDs in order to avoid dangerous over-treatment in patients with co-existing widespread pain. Effective pain management is a primary goal of patient care, although it has not been investigated in detail in patients with SSc.
\end{abstract}

๑) 2015 Elsevier Ltd. All rights reserved.

\footnotetext{
* Corresponding author. Department of Internal Medicine and Medical Specialities, Rheumatology Unit, Sapienza University of Rome, 00161 Roma, Italy. Tel.: +39 02 39042489; fax: +39 0239043454.

E-mail address: manuela.difranco@uniroma1.it (M. Di Franco).

1 MDF and LB contributed equally to drafting the manuscript.
} 


\section{Introduction}

Connective tissue diseases (CTDs) are characterised by multiple symptoms generally related to organ injury. One of the most frequent is pain, the perception and threshold of which may be influenced by many biological, psychological and social factors interacting with the central and peripheral nervous systems. It may be acute or chronic: acute pain is often primarily attributable to inflammation and/or damage to peripheral structures (i.e. nociceptive input), whereas chronic pain (generally defined as lasting $\geq 3$ months) is more likely to be due to input from the central nervous system (CNS). The chronic nature of CTDs such as systemic lupus erythematosus (SLE) and systemic sclerosis (SSc), which are often associated with pain and stress, can also trigger widespread chronic pain conditions such as fibromyalgia (FM).

\section{Pain in systemic sclerosis}

Systemic sclerosis (SSc, also known as scleroderma) is a severe rheumatic condition characterised by skin thickening and internal organ fibrosis [1] that is classically classified as limited cutaneous SSc (lcSSc), which has rare organ involvement, and diffuse cutaneous SSc (dcSSc), which has a worse prognosis and is characterised by rapid fibrosis [1,2].

Pain is a ubiquitous problem in SSc, and may be caused by digital ulcers, Raynaud's phenomenon, skin breakdown, joint contractures and/or gastrointestinal (GI) disrorders [3]. However, despite its impact on the patients' quality of life, it has not been widely studied. In a large study published by the Canadian Scleroderma Research Group, 85\% of the 585 patients reported pain, which is correlated with more frequent episodes of Raynaud's phenomenon, active ulcers, worse synovitis, and gastrointestinal symptoms [4]; other authors have reported similar findings and that they correlate with a poor quality of life [5-7].

\section{SSc and Raynaud's phenomenon}

Raynaud's phenomenon (RP) is the most frequent and earliest manifestation of SSc. It is caused by digital vasospasms usually triggered by exposure to cold or stress, which lead to the three phases of the classical colour change from white to blue (cyanosis) and then red (erythema), and is frequently associated with pain and sometimes with paresthesia, numbness and impaired hand function. It can be effectively treated by various classes of drugs, whose benefits include a reduction in the frequency and severity of attacks, and the prevention and/or healing of digital ulcers. The first-line non-pharmacological treatment of Raynaud's phenomenon involves avoiding or minimising exposure to cold, the use of warm gloves, and avoiding aggravating factors such as smoking and certain drugs, although these measures are more effective in the case of primary rather than secondary Raynaud's phenomenon. Pharmacological measures usually start with calcium channel blockers but, if these are ineffective, other options include topical nitroglycerin, and alpha adrenergic or angiotensin receptor antagonists. Intravenous prostacyclin analogues are warranted in severe cases, particularly if there is a threat of digital ischaemia, but they are expensive and, as they burdened by substantial risks (including the induction of severe hypotension), close monitoring is required during their administration. Novel approaches include the use of endothelin receptor antagonists, phosphodiesterase inhibitors and statins, although their place in the therapeutic armamentarium remains to be established, and it may also be possible to combine drugs acting on different target mechanisms, although this may be limited by questions of cost.

Finally, surgical approaches (particularly thoracic sympathectomy) have fallen out of favour, probably because of improvements in pharmacological treatments [8].

\section{SSc and digital ulcers}

Often persistent and recurrent digital ulcers are one of the most frequent and burdensome clinical manifestations, and occur in more than $50 \%$ of patients. They may simultaneously affect more than one 
finger, and lead to severe pain and function limitations [9]. The lack of validated guidelines has prompted a number of researchers to seek the best treatment, and a very recent study published by Giuggioli et al. tested the initial use of local lidocaine and prilocaine ( $25 \mathrm{mg}$ of both per gram of $5 \%$ EMLA cream), followed by local and oral morphine depending on the severity of the pain as measured by means of a $10 \mathrm{~cm}$ visual analogue scale (VAS), and found that the deep wound debridement crucial for healing was better tolerated [10].

\section{SSc and synovitis}

Between $40 \%$ and $80 \%$ of SSc patients complain of musculoskeletal pain, which is more problematic in patients with early diffuse SSc. The pain may not be sufficiently localised to attribute it to a particular anatomical location, but a number of pain syndromes have been identified.

1. Tendinitis: Tendon friction rubs mainly affect patients with early diffuse SSc. They have a frequency of $23-65 \%$, but this tends to decline over time [11,12]. They are considered to be associated with more active disease and worse outcomes.

2. Polyarthritis: Between $36 \%$ and $80 \%$ of patients complain of polyarthralgias, which may be more frequent in those with early SSc, although some studies have found their occurrence more equally distributed between limited and diffuse SSc [13]. The wide range of articular and non-articular changes observed in radiographs of SSc patients go from juxta-articular osteoporosis and joint space narrowing to frank erosions in the metacarpophalangeal (MCP), proximal interphalangeal (PIP), and distal interphalangeal (DIP) joints, and wrist. It has been said that bony erosions (mainly in the hands) affect $4-57 \%$ of patients who have had SSc for seven years, and joint space narrowing affects 16-92\% [14]; however, concern has been raised that some of the joint space narrowing may be related to concomitant osteoarthritis and not just SSc.

3. Rheumatoid arthritis (RA): The recent availability of anti-cyclicitrullinated peptide (CCP) assays has led to the finding that $1-15 \%$ of SSc patients have overlapping anti-CCP antibody-positive RA. However, it should be noted that anti-CCP antibodies alone do not define RA, and it is not known how many SSc patients without RA are anti-CCP positive.

4. Fibromyalgia (FM): Studies reported that $48 \%$ of patients had 11 or more tender points (TPs), whereas the mean TP count was 7 (of 18) in the Malcarne study [15]. Clinical experience suggests that FM is not uncommon in patients with SSc or other CTDs, and dedicated work is needed in this field, including studies using the 2010 fibromyalgia criteria.

\section{SSc and gastrointestinal disorders}

The gastrointestinal (GI) is the second most frequently involved organ system in SSc patients [16], who often experience complications such as gastro-esophageal symptoms, abdominal pain and distension, weight loss and nutritional deficiencies, diarrhea, incontinence, and constipation.

The esophagus is the most frequently affected part of the GI tract, and up to $90 \%$ of patients describe symptoms of heartburn, regurgitation and dysphagia. These dysfunctions are probably due to smooth muscle atrophy (particularly the inner circular layer of the muscularis propria) and fibrosis affecting the distal two-thirds of the esophagus but sparing the proximal part that causes the loss of normal neural function. Lifestyle modifications and the avoidance of exacerbating food groups are often suggested first, but patients often need intensive treatment with proton pump inhibitors to control their symptoms.

Up to 50\% of SSc patients report early satiety, nausea, bloating, and abdominal discomfort. The pathophysiology is not clear but it is possible that lymphocyte activation plays an important role in causing smooth muscle atrophy and collagen deposition, leading to severe ultrastructural alterations in smooth muscle cells and nerve fibres. It is thought that gut dysfunction relates to a neuropathic process in SSc patients [16]. 
The clinical management of gastric motility disorders can be difficult because of their poor correlations with symptoms. Dietary modifications with the addition of a prokinetic agent is often the mainstay of treatment, and probiotics may be useful in some patients. The use of metoclopramide can improve gastric motility and motor activity, and somatostatin analogues such as octreotide have also been used to induce contractile activity throughout the bowel. It has been reported that up to $18 \%$ of patients with SSc are at high risk of malnutrition due to perioral sclerosis, esophageal dysmotility and abdominal discomfort; the management of weight loss and malnutrition requires a multidisciplinary team approach in which dieticians, nutrition specialists and ward nursing staff play a crucial role.

Diarrhea can affect up to $50 \%$ of patients, who need to be fully assessed because the cause is multifactorial. Once the contributory causes of malabsorption have been investigated, symptomatic approaches such as dietary measures to increase stool consistency and use of loperamide to inhibit peristalsis and secretion can be tried; however, caution is required in order to avoid pseudoobstruction. Cholestyramine or other bile salt acid sequestrants may be helpful [16].

The colon and anorectum are the second most frequently affected parts of the GI tract, and it has been suggested that the anorectal dysfunction reported by $50-70 \%$ of SSc patients is due to neuronal dysfunction, smooth muscle atrophy and fibrosis affecting the internal anal sphincter. Fecal urgency can arise because of reduced rectal compliance and capacity due to collagen deposition, and fecal incontinence has a significantly negative impact on the patient's quality of life. Practical specialist management such as biofeedback and bowel and pelvic floor muscle training can be offered although the evidence is limited. Surgical repair of the anal sphincter has been attempted but the long-term outcomes suggest worsening of continence and so this approach is not generally advocated [16].

It has been reported that colonic involvement occurs in $20-50 \%$ of patients, who often lack the post-prandial gastrocolic response mediated by the cholinergic pathway, thus reducing colonic motility, prolonging colonic transit and leading to constipation. Unfortunately, laxatives frequently offer little benefit: stimulant laxatives rely on contact with the bowel mucosa, which is unpredictable, and osmotic laxatives can aggravate bloating and discomfort. It has been shown that the 5HT4 receptor agonist prucalopride accelerates colonic transit but, although the results have been promising, they have only been published in case reports. Opioid antagonists such as methylnaltrexone do not seem to be very beneficial in patients with SSc because of the nature of their bowel dysmotility. Biofeedback training is useful in the case of idiopathic constipation, but it has not been studied in SSc. There are no published data corning the effect of sacral nerve stimulation (SNS) on constipation in SSc patients, although it is useful in idiopathic constipation; however, the drawbacks of SNS are that it is an expensive invasive procedure associated with the risks of infection, lead migration and pain.

Intestinal pseudo-obstruction is a rare GI manifestation of SSc. The treatment algorithms mention professional patient counselling, and depressive symptoms have been reported to be associated with GI involvement in SSc patients. Treating gastroenterologists should take an overall holistic approach and their patients' quality of life, functional status and depressive symptoms, whereas treatment interventions for SSc are limited [16].

\section{Pain in systemic lupus erythematosus}

\section{SLE and inflammatory pain}

Inflammation is the most frequent cause of pain in SLE patients. It is generally due to inflammatory arthritis, which is included in the clinical set of the American College of Rheumatism (ARC) classification criteria $[17,18]$. The arthritis is typically not erosive, does not induce joint deformity, and frequently precedes the other manifestations of SLE. It is associated with morning stiffness for more than $30 \mathrm{~min}$, can be evanescent or persistent, affects the knees and the small joints of hands (PIPs), and produces objective evidence of inflammation (tenderness, swelling and effusion). A minority of patients may show deforming reducible joint involvement of the hands (Jaccoud's arthropathy) [19]. The presence of synovitis is due to the production of cytokines such as interleukin (IL)-6, IL-17, interferon (INF) alpha, IL-18, tumour necrosis factor (TNF) and B cell stimulating factor (BSF)-2, which 
main responsible for immune response attivation and tissue damage [20]. The clincal set of the ACR criteria includes serositis, which may present as painful or painless pleural or pericardial effusion and ascitis as a result of inflammation of the lining of lung, heart, and abdominal structures. Abdominal pain is reported by $8-40 \%$ of SLE patients but may also be due to other causes, including mesenteric vasculitis and pancreatitis $[21,22]$.

\section{SLE and neuropathic pain}

SLE patients show a wide range of central nervous system (CNS) manifestations, including neuropsychiatric disorders and syndromes associated with the presence of auto-antibodies [23]. Although its relationship with SLE is not clear, headache and migraine are reported by $32-66 \%$ of SLE patients and may have various causes, including neuropsychiatric SLE (NPSLE) [24]. A recent study of 40 SLE patients found that $70 \%$ experienced headache (tension type headache in $37.5 \%$, migraine in $30 \%$, cluster headaches in $2.5 \%$, and intracranial hypertension in $5 \%$ ) but there was no association with disease activity $[25,26]$. A close association with cognitive impairment, depression, pain and fatigue has been found in NPSLE patients, but the underlying causes are unclear. Auto-antibodies cross-reacting with DNA, $N$-methyl-D-aspartate receptors, and anti-endothelial and anti-phospholipid antibodies are the most common factors associated with the pathogenesis of NPSLE [27].

It has been shown that peripheral neuropathies may be equally or even more frequent than some CNS syndromes in SLE patients [28,29], and therefore another source of pain. A recent long-term study of more than 2000 patients found that the prevalence of peripheral neuropathies was $5.9 \%$, and that $66.7 \%$ of these were peripheral neuropathies due to SLE, of which sensory and sensorimotor axonal polyneuropathies were the most frequent. Small-fibre neuropathies and demyelinating polyneuropathies are other causes of peripheral neuropathy in SLE [30].

Neuropathic pain can be also a consequence of herpes zoster (HZ) infection, a painful neurocutaneous disease caused by the reactivation of varicella zoster virus. Immunological studies of SLE patients have shown abnormal T cell-mediated cytotoxicity, and the suppression of cellular immunity may be involved in the pathogenesis of virus reactivation [31]. It is known that disease activity and the use of corticosteroids and/or immunosuppressive therapies contribute to $\mathrm{HZ}$ infection, although a study of a large cohort of SLE patients showed an annual HZ incidence rate of 6.4 events/1000 patientyears without any association with disease activity (SLEDAI $<8$ ); post-herpetic neuralgia was detected in $19 \%$ of the patients [32].

\section{SLE and central pain}

Musculoskeletal pain is reported by 50-90\% SLE of patients during the course of the disease [33] and chronic widespread pain, which affects $5-10 \%$ of the general population [34], by $65-80 \%$ of SLE patients $[35,36]$. Until a few years ago, the pain associated with many rheumatic diseases was considered to be peripheral in origin and induced by the well-known mechanisms of acute or chronic inflammation, or morpho-structural alterations in the involved joints [37,38]. However, the mechanisms underlying chronic widespread pain (the prototype of which is FM) have only recently been identified as neurophysiological modifications in the perception, transmission and, above all, processing of nociceptive afferents at the level of the CNS, which seem to be caused by what has come to be called "sensitisation": i.e. a permanent state of neuronal hyperexcitability that involves all of the peripheral and central structures of the nociceptive system and causes hyperalgesia and allodynia $[39,40]$. Immunological cascades may play a role in maintaining central sensitivity and chronic pain, which is increased when CNS glial cells release pro-inflammatory cytokines; the traditional dichotomy of inflammatory $v s$ non-inflammatory pain may therefore be less appropriate than previously thought [41]. The neurophysiological mechanisms underlying central sensitisation syndromes may also play a role in causing the painful symptoms characterising CTDs. Patients with chronic pain conditions are generally female and have experienced an early-life trauma or have a personal or family history of chronic pain, or a personal history of other centrally mediated symptoms (insomnia, fatigue, cognitive alterations and mood disturbances) and cognitions such as catastrophising, all of which can predict the likelihood that acute pain will become chronic [42,43]. 


\section{SLE and fibromyalgia}

The clinical hallmark of FM is chronic widespread pain and tenderness to palpation of at least 11/18 tender points (TPs) [44]. Various symptoms are characteristically associated with FM, such as sleep, mood and neurocognitive disorders, as indicated by the 2010 ACR classification criteria [45]. The estimated prevalence of FM in the general population is about 1-3\% in different groups [46], and many studies have investigated its prevalence in SLE patients, and evaluated how concomitant FM can influence the symptoms and the activity of SLE. Morand et al. [47] found a $25.3 \%$ prevalence of FM in a cohort of 87 SLE patients; Middleton et al. [48] a 22\% prevalence in a group of 102 patients; and Iannuccelli et al. [36] a prevalence of $33 \%$ in a cohort of 50 patients. The co-existence of FM may make it difficult to make a differential diagnosis with SLE flares.

The most widely used indices for objectively measuring SLE disease activity are the British Isles Lupus Assessment Group (BILAG) Index, the European Consensus Lupus Activity Measurement (ECLAM), and the Systemic Lupus Erythematosus Disease Activity Index (SLEDAI), but none of these rates pain as such [49]. The Systemic Lupus International Collaborating Clinics (SLICC) group has recently proposed a new set of criteria that includes the specific clinical manifestations frequently reported by SLE patients. The features of arthritis are specified because of the overlap between FM and SLE in some patients and it is necessary to confirm that there is joint line tenderness and not more diffuse allodynia. It is also necessary to point out that the cause of all the SLICC criteria is attributable to SLE and not to another disease process or condition [50]. Middleton et al. [48] found that SLE patients with concomitant FM had significantly more frequent and severe symptoms, but there were no significant difference in SLE activity measures between the patients with or without FM, and other authors have confirmed the absence of correlations between concomitant FM and disease activity, joint damage or organ dysfunction [50,51].

Fatigue is another symptom characterising FM and the most common constitutional and debilitating symptom associated with pain in SLE patients [52], with prevalence rates of 50-80\% [53]. Many studies have failed to demonstrate a correlation between fatigue and SLE disease activity, and only a few have observed greater disease activity in patients reporting fatigue [36,53,54]. Moreover, SLE patients with overlapping FM report symptoms such as headache, morning stiffness, diffuse alopecia, and arthralgia significantly more often [55].

The health-related quality of life (HRQoL) of SLE patients can be evaluated by administering questionnaires such as the Short-form 36 (SF-36) [56], and the Patient Acceptable Symptom State (PASS) a simple questionnaire used to assess well-being in various rheumatic diseases including SLE [57].

Pain is associated with anxiety and depression, and perceptions of reduced physical functioning, and so SLE patients need psychosocial interventions to relieve their pain and distress and improve their coping skills [58]. Pain coping cognitions such as self-efficacy for pain control and pain catastrophising can influence symptoms such as pain, stiffness, fatigue, and psychological distress in SLE patients. Recent data demonstrate that patients with low levels of self-efficacy for pain control and/or high levels of pain catastrophising report more physical symptoms and psychological distress, and highlight the importance of assessing coping constructs in SLE patients [59]. Regardless of FM, pain influences the quality of life, and SLE patients who have higher pain levels also report stiffness and fatigue. SLE patients frequently report symptoms such as pain, fatigue and musculoskeletal distress, all of which are related to low HRQoL scores [60] and cause functional limitations that lead to a significant economic burden. The working productivity of SLE patients with the highest levels of pain is lower than that of SLE patients with less pain [61]. Consequently increased work absenteeism and work disability (WD) rates have been observed in numerous SLE studies. The reported WD rates range from $20 \%$ to $50 \%$, and vary widely among SLE population studies. The demographic factors associated with WD include age, a low educational level, low socio-economic status and race, and correlations have been found between WD and pain, fatigue, depressive symptoms, comorbidities, disease duration and activity, joint damage and cognitive dysfunction [62]. The differential diagnosis of SLE and FM may be difficult because the two diseases have some symptoms in common, and low antinuclear antibody (ANA) titres are frequently reported in the general population and FM patients, thus making it necessary to evaluate patients clinically in order to avoid misdiagnoses.

Although secondary FM is not associated with SLE disease activity, it may worsen the quality of life of patients with SLE, and requires appropriate treatment. It is necessary to assess whether pain in SLE 
patients is localised or widespread, visceral or musculoskeletal. SLE activity should be measured, and treatment with immunosuppressive drugs and steroids should be optimised on the basis of the severity of the disease $[63,64]$. The correct interpretation of the FM symptoms is crucial to avoid overtreatment because concomitant FM can simulate SLE flares. Once it has been excluded that the pain is attributable to disease activity, the same treatment as that used for FM can be administered. Analgesic drugs such as acetaminophen or tramadol can be safely used in patients with widespread pain and SLE [65]. Anti-epileptic drugs such as gabapentin and pregabalin have been approved for the treatment of neuropathic pain: both drugs have favourable safety and tolerability profiles [66], and pregabalin has also been approved by the American Food and Drug Administration (FDA) for FM. Low doses of tricyclic antidepressants are useful in the case of musculoskeletal pain and headache. Selective serotonin and serotonin norepinephrine reuptake inhibitors such as duloxetine (approved by the FDA for FM) can be used in SLE patients with pain and depression [67].

\section{Summary}

Pain is frequently associated with CTDs. The pain in SLE can have various causes (e.g. inflammatory, neuropathic and central pain), but inflammatory joint pain is one of the most frequent. Chronic widespread pain is the cornerstone of FM, and many studies have investigated the prevalence of FM in SLE patients, and evaluated how concomitant FM has a bearing on SLE symptoms and disease activity. The treatment of pain in SLE patients requires a differential diagnosis; in particular, it is necessary to establish whether the pain is localised or widespread, visceral or musculoskeletal. The first step is to evaluate SLE activity, and optimise specific SLE treatment with immunosuppressive drugs and steroids. FM symptoms in an SLE patient may be misinterpreted as lupus disease activity and thus lead to over-treatment: when it has been excluded that SLE activity is the main cause of pain, the treatment is the same as that used for FM. Analgesic drugs such as acetaminophen or tramadol can be safely used in patients with widespread pain and SLE. In many cases, non-pharmacological treatments such as aerobic exercise and cognitive behavioural therapy may also be useful.

Pain is a virtually ubiquitous problem in SSc: $83 \%$ of patients in a recent large sample reported significant pain. Early in the disease process, patients report non-specific muscle pain and stiffness, whereas other symptoms (e.g. difficulty in swallowing and gastrointestinal discomfort) emerge as the disease progresses. Effective pain management is a primary goal of patient care, although it has not been investigated in detail in patients with SSc.

\section{Disclosure of interest}

The authors declare that they have no conflict of interest concerning this article.

\section{Practice points}

- Pain is a cornerstone of the definition of SLE and SSc, but it may be due to a mechanism related to central pain sensitisation (similar to that observed in FM) and inflammation.

- Inflammatory pain symptoms can be reduced by NSAIDs and DMARDs, but many patients continue to experience moderate pain due to alterations in central pain regulation mechanisms, as in the case of CWP.

- It is important to identify the symptoms of CWP in order to be able to manage and treat patients with CTDs appropriately.

- Effective pain management is a primary goal of patient care, although it has not been investigated in detail in patients with SSc and SLE.

- Researchers and clinicians should be encouraged to assess perceived physical health, health worries, mental health, and social support, in addition to routinely evaluating organ disease severity in SSc and SLE patients. 


\section{Research agenda}

- To develop new laboratory and clinical indices for distinguishing CWP from inflammatory pain in CTDs in order to reduce misdiagnoses.

- To evaluate the adequacy and appropriateness of measures for diagnosing inflammatory and central pain.

- To determine whether new instrumental methods such as ultrasonography can distinguish CWP from other types of pain.

- To develop new recommendations for differentiating widespread pain in the context of CTDs.

- To promote future multicentre studies and registries of widespread pain in CDTs in order to reduce the overestimated disease activity.

\section{References}

[1] Medsger TA. Natural history of systemic sclerosis and the assessment of disease activity, severity, functional status, and psychologic well-being. Rheum Dis Clin North Am 2003;29:255-73.

[2] LeRoy EC, Black C, Fleischmajer R, et al. Scleroderma (systemic sclerosis): classification, subsets and pathogenesis. J Rheumatol 1988;15:202-5.

[3] Merkel PA. Measurement of functional status, self-assessment, and psychological well-being in scleroderma. Curr Opin Rheumatol 1998;10:589-94.

*[4] Schieir O, Thombs BD, Hudson M, et al., Canadian Scleroderma Research Group. Prevalence, severity, and clinical correlates of pain in patients with systemic sclerosis. Arthritis Care Res (Hoboken) 2010;62(3):409-17.

*[5] Benrud-Larson LM, Haythornthwaite JA, Heinberg LJ, et al. The impact of pain and symptoms of depression in scleroderma. Pain 2002;95:267-75.

[6] Richards HL, Herrick AL, Griffin K, et al. Systemic sclerosis: patients' perceptions of their condition. Arthritis Rheum 2003; 49:689-96.

[7] Suarez-Almazor ME, Kallen MA, Roundtree AK, et al. Disease and symptom burden in systemic sclerosis: a patient perspective. J Rheumatol 2007;34:1718-26.

[8] Sinnathurai P, Schrieber L. Treatment of Raynaud phenomenon in systemic sclerosis. Intern Med J 2013;43(5):476-83.

*[9] Guillevin L, Hunsche E, Denton CP, et al., DUO Registry Group. Functional impairment of systemic scleroderma patients with digital ulcerations: results from the DUO Registry. Clin Exp Rheumatol 2013 Mar-Apr;31(2 Suppl. 76):71-80.

[10] Giuggioli D, Manfredi A, Vacchi C, et al. Procedural pain management in the treatment of scleroderma digital ulcers. Clin Exp Rheumatol 2015;33:5-10.

[11] Ostojić P, Damjanov N. Different clinical features in patients with limited and diffuse cutaneous systemic sclerosis. Clin Rheumatol 2006;25(4):453-7.

[12] Khanna PP, Furst DE, Clements PJ, et al., D-Penicillamine Investigators. Tendon friction rubs in early diffuse systemic sclerosis: prevalence, characteristics and longitudinal changes in a randomized controlled trial. Rheumatology (Oxford) 2010;49:955-9.

[13] Brower LM, Poole JL. Reliability and validity of the Duruoz Hand Index in persons with systemic sclerosis (scleroderma). Arthritis Rheum 2004;51:805-9.

[14] Koutaissoff S, Vanthuyne M, Smith V, et al. Hand radiological damage in systemic sclerosis: comparison with a control group and clinical and functional correlations. Semin Arthritis Rheum 2011;40:455-60.

[15] Malcarne VL, Hansdottir I, McKinney A, et al. Medical signs and symptoms associated with disability, pain, and psychosocial adjustment in systemic sclerosis. J Rheumatol 2007;34:359-67.

[16] Hansi H, Thoua N, Carulli M, , et alConsensus Best Practice pathway of the UK Scleroderma Study Group. Gastrointestinal manifestations of systemic sclerosis. Clin Exp Rheumatol 2014;32:S214-21.

*[17] Hochberg MC. Updating the American College of Rheumatology revised criteria for the classification of systemic lupus erythematosus. Arthritis Rheum 1997;40:1725.

[18] Petri M, Orbai AM, Alarcón GS, et al. Derivation and validation of the Systemic Lupus International Collaborating Clinics classification criteria for systemic lupus erythematosus. Arthritis Rheum 2012;64:2677-86.

[19] Askanase A, Shum K, Mitnick H. Systemic lupus erythematosus: an overview. Soc Work Health Care 2012;51:576-86.

[20] Yap DY, Lai KN. The role of cytokines in the pathogenesis of systemic lupus erythematosus- from bench to bedside. Nephrology 2013;18:243-55.

[21] Kwok SK, Seo SH, Ju JH, et al. Lupus enteritis: clinical characteristics, risk factor for relapse and association with anti-endothelial cell antibody. Lupus 2007; 16:803-9.

[22] Yuan S, Lian F, Chen D, et al. Clinical features and associated factors of abdominal pain in systemic lupus erhitematosus. J Rheumatol 2013;40:2015-22.

[23] ACR Ad Hoc Committee on Neuropsychiatric Lupus Nomenclature. The American College of Rheumatology nomenclature and case definitions for neuropsychiatric lupus syndromes. Arthritis Rheum 1999;42:599-608.

[24] Sfikakis PP, Mitsikostas DD, Manoussakis MN, et al. Headache in systemic lupus erythematosus: a controlled study. Br J Rheumatol 1998;37:300-3.

[25] Badry R, Gamal RM. Different types of headache in patients with systemic lupus erythematosus. Int J Neurosci 2014;9. 
[26] Glanz BI, Venkatesan A, Schur PH, et al. Prevalence of migraine inpatients with systemic lupus erythematosus. Headache $2001 ; 41: 285-9$

[27] Conti F, Alessandri C, Perricone C, et al. Neurocognitivedysfunction in systemic lupus erythematosus:association with antiphospholipid antibodies,disease activity and chronic damage. PLoS One 2012;7:33824.

*[28] Hanly JG, Urowitz MB, Su L, et al., for the Systemic Lupus International Collaborating Clinics (SLICC). Prospective analysis of neuropsychiatric events in an international disease inception cohort of patients with systemic lupus erythematosus. Ann Rheum Dis 2010;69:529-35.

[29] Hanly JG, Urowitz MB, Siannis F, et al., for the Systemic Lupus International Collaborating Clinics. Autoantibodies and neuropsychiatric events at the time of systemic lupus erythematosus diagnosis: results from an international inception cohort study. Arthritis Rheum 2008;58:843-53.

[30] Oomatia A, Fang H, Petri M, et al. Peripheral neuropathies in systemic lupus erythematosus. Arthritis Rheum 2014;66: $1000-9$.

[31] Nagasawa K, Yamauchi Y, Tada Y, et al. High incidence of herpes zoster inpatients with systemic lupus erythematosus: an immunological analysis. Ann Rheum Dis 1990;49:630-3.

[32] Borba EF, Ribeiro ACM, Martin P, et al. Incidence, risk factors, and outcome of herpes zoster in systemic lupus erythematosus. JCR: J Clin Rheumatol 2010;16.

[33] Waldheim E, Elkan AC, Pettersson S, et al. Health-related quality of life, fatigue andmood in patients with SLE and high levels ofpain compared to controls and patients with low levels of pain. Lupus 2013;22:1118-27.

*[34] Sarzi-Puttini P, Atzeni F, Mease P. Chronic wide spread pain or fibromyalgia? That is the question. Best Pract Res Clin Rheumatol 2011;25:131-2.

[35] Wolfe F, Petri M, Alarcón Gs, et al. Fibromyalgia,systemic lupus erythematosus (SLE),and evaluation of SLE activity. J Rheumatol 2009;36:82-8.

[36] Iannuccelli C, Spinelli FR, Guzzo MP, et al. Fatigue and widespread pain in systemic lupus erythematosusand Sjögren's syndrome: symptoms of the inflammatory disease or associated fibromyalgia? Clin Exp Rheumatol 2012;30:117-21.

[37] Yunus MB. Fibromyalgia and overlapping disorders: the unifying concept of central sensitivitysyndromes. Semin Arthritis Rheum 2007;36:339-56.

*[38] Cazzola M, Atzeni F, Boccassini L, et al. Physiopathology of pain in rheumatology. Reumatismo 2014;66:4-13.

[39] Yunus MB. Role of central sensitization in symptoms beyond muscle pain, and the evaluation of a patient with widespread pain. Best Pract Res Clin Rheumatol 2007;21:481-97.

[40] Kindler LL, Bennett RM, Jones KD. Central sensitivity syndromes: mounting pathophysiologic evidence to link fibromyalgia with other chronic pain disorders. Pain Manag Nurs 2011;12:15-24.

*[41] Clauw DJ, Witter J. Pain and rheumatology thinking outside the joint. Arthritis Rheum 2009;60:321-4.

[42] Kazis LE, Meenan RF, Anderson JJ. Pain in the rheumatic diseases. Investigation of a key health status component. Arthritis Rheum 1983;26:1017-22.

[43] Steiman AJ, Pope JE, Thiessen-Philbrook H, et al. Non-biologic disease-modifying antirheumatic drugs (DMARDs) improve pain in inflammatoryarthritis (IA): a systematic literature review of randomized controlled trials. Rheumatol Int 2013;33: 1105-20.

[44] Wolfe F, Smythe HA, Yunus MB, et al. The American College of Rheumatology 1990 criteria for the classification of fibromyalgia: report of theMulticenter Criteria Committee. Arthritis Rheum 1990;33:160-72.

[45] Wolfe F, Clauw DJ, FitzCharles MA, et al. The American College of Rheumatology preliminary diagnostic criteria for fibromyalgia and measurement of symptom severity. Arthritis Care Res (Hoboken) 2010;62:600-10.

*[46] Jones GT, Atzeni F, Beasley M, et al. The prevalence of fibromyalgia in the general population: a comparison of the American College of Rheumatology 1990, 2010, and Modified 2010 classification criteria. Arthritis Rheum 2015;67: $568-75$.

[47] Morand EF, Miller MH, Whittingham S, et al. Fibromyalgia syndrome and disease activity in systemic lupus erythematosus. Lupus 1994;3:187-91.

[48] Middleton GD, Mcfarlin JE, Lipsky PE. The prevalence and clinical impact of fibromyalgia in systemic lupus erythematosus. Arthritis Rheum 1994;37:1181.

[49] Di Franco M, Guzzo MP, Spinelli FR, et al. Pain and systemic lupus erythematosus. Reumatismo 2014;66:33-8.

[50] Gladman DD, Urowitz MB, Gough J, et al. Fibromyalgia is a major contributor to quality of life in lupus. J Rheumatol 1997; 24:2145-8.

[51] Friedman AW, Tewi MB, Ahn C, et al. Systemic lupus erythematosus in three ethnic groups:XV. Prevalence and correlates of fibromyalgia. Lupus 2003;12:274-9.

*[52] Petri M, Kawata AK, Fernandes AW, et al. Impaired health status and the effect of pain and fatigue on functioning in clinical trial patients with systemic lupus erythematosus. J Rheumatol 2013;40:1865-74.

[53] Tench CM, McCurdie I, White PD, et al. The prevalence and associations of fatigue in systemic lupus erythematosus. Rheumatology (Oxford) 2000;39:1249-54.

[54] Da Costa D, Dritsa M, Bernatsky S, et al. Dimensions of fatigue in systemic lupus erythematosus: relationship to disease status and behavioral and psychosocial factors. J Rheumatol 2006;33:1282-8.

[55] Gräfe A, Wollina U, Tebbe B, et al. Fibromyalgia in lupus erythematosus. Acta Derm Venereol 1999;79:62-4.

[56] Griffiths B, Mosca M, Gordon C. Assessment of patients with systemic lupus erythematosus and the use of lupus disease activity indices. Best Pract Res Clin Rheumatol 2005;19:685-708.

[57] Conti F, Ceccarelli F, Massaro L, et al. Evaluation of the patient acceptable symptom state (PASS) in Italian patients affect by systemic lupus erythematosus: association with disease activity indices. PLoS One 2013;8:e73517.

[58] Greco CM, Rudy TE, Manzi S. Adaptation to chronic pain in systemic lupus erythematosus: applicability of the multidimensionalpain inventory. Pain Med 2003;4:39-50.

[59] Somers T], Kurakula PC, Criscione-Schreiber L, et al. Self-efficacy and pain catastrophizing in systemic lupus erythematosus: relationship to pain, stiffness, fatigue, and psychological distress. Arthritis Care Res (Hoboken) 2012;64:1334-40.

[60] Pettersson S, Lövgren M, Eriksson LE, et al. An exploration of patient-reported symptoms in systemic lupus erythematosus and the relationship to health-related quality of life. Scand J Rheumatol 2012;41:383-90. 
[61] Clarke AE, Bloch DA, Danoff DS, et al. Decreasing costs and improving outcomes in systemic lupus erythematosus: using regression trees to develop health policy. J Rheumatol 1994;21:2246-53.

[62] Utset TO, Baskaran A, Segal BM, et al. Work disability, lost productivity and associated risk factors in patientsdiagnosed with systemic lupus erythematosus. Lupus Sci Med 2015;2:e000058.

[63] Bertsias G, Ioannidis JP, Boletis J, et al. EULAR recommendations for the management of systemic lupus erythematosus. Report of a Task Force of the EULAR Standing Committee for International Clinical Studies Including Therapeutics. Ann Rheum Dis 2008;67:195-205.

[64] Conti F, Ceccarelli F, Massaro L, et al. Biologicaltherapies in rheumatic diseases. Clin Ther 2013;164:e413-428.

[65] Bennett RM, Schein J, Kosinski MR, et al. Impact of fibromyalgia pain on health-related quality of life before and after treatment with tramadol/acetaminophen. Arthritis Rheum 2005;53:519-27.

[66] Spaeth M. Is pregabalin a safe and effective treatment for patients with fibromyalgia? Nat Clin Pract Rheumatol 2008;4: 514.

[67] Mease PJ, Dundon K, Sarzi-Puttini P. Pharmacotherapy of fibromyalgia. Best Pract Res Clin Rheumatol 2011;25:285-97. 